\title{
Editorial
}

\section{Technology Advancement and Research Progress in Orthopedic Surgery}

\author{
Luminita Labusca*,§
}

\begin{abstract}
$1^{\text {st }}$ Independentei Boulevard, University Hospital Saint Spiridon Iasi Romania, Orthopedic and Traumatology Clinic, SBIM Systems, Bioinformatics and Modelling, Frankfurt, Germany
\end{abstract}

Undoubtefully, orthopedics is one of the most technical surgical specialities. Modern orthopedic surgery took shape in the process of making use of the advanced science and the latest technological achievments of the time. It was and, in some respect, still continues to be, a story of clinical driven discovery placed in the forefront of science by the inquiring minds of orthopedic surgeons, in their quest for answering stringent clinical demands. In the mid 1960s Charnley introduced the hip prosthesis and the use of bone cement for implant fixation, aiming to treat the pain and disability produced by cartilage deterioration in advanced stages of osteoarthritis [1]. Based on Chrnaley extensive knowledge and contibution in tribology, implant development was possible due flourishing metalurgy and polymer technology. Total joint replacement was than implemented and is continously refined today. Increasing surgeon expertise, follow up data gathered by means of national joint replacement registries as well as technological optimization are only few of the factors contributing to the wide spread of the procedure, Hard - hard friction couples, trabecular technology or biological coatings are continously improving the quality and lifetime of available prosthesis. Joint reconstructive surgery is nowadays the most succesful intervention in all times and all surgical specialities in terms of cost efficiency and patient satisfaction [2] In the middle $60 \mathrm{~s}$, when RW Jhonson and R.O Connor were taking the bulky video camera on their shoulder to inspect joints, it was an attempt to elucidate and document tricky cases of knee pathology. Trained by the pioneer of arthroscopy, Masuki Watanabe, they were setting the basis of a new diagnostic and operative method which was based on the optics and electronics technology. The technical improvment introduced by the digitalization of video capture, transmission and storage, has made possible the establishment of artroscopic surgery as a precise, minimal invasive procedure. A new range of possibilities in treating ligament meniscal and cartilage injuries therefore occured, allowing for restaurative approaches and improved postinterventional functional recovery. Torn cruciate ligaments in the knee or recurrent shouder dislocation are no longer a career end for sport professional due to the widespread of reconstructive

\footnotetext{
*Address correspondence to this author at the Independentei Boulevard University Hospital, Saint Spiridon Iasi, Romania;

Tel: +40749162219; Fax: +49-069-706212;

E-mails: luminitasimion@yahoo.com, luminita.labusca@online.de

${ }^{\S}$ Guest Editor
}

arthroscopic surgery. Degenerative rotator cuff meniscal or cartilage lesions can be treated without the inconvenient open procedure. Arthroscopy continues to enrich knowledge about so far obscure joint or periarticular symptoms. Giaccommo et al. are reporting a unique case serial of bilateral suprascapular nerve entrapment produced by labral ganglion cyst. Complete functional recovery was achieved after arthroscopic nerve decompresion and labral tear repair allowing for patient's complete social and professional reintegration. In his own laboratory, M Ulrist, orthopedic surgeon and passionate in biochemistry observed the demineralized bone fragments induced ectopic bone formation in rabbit muscle [3]. The discovery of Bone Morphogenetic Protein, BMP, opened a new chapter in biology. A plethora of growth factors involved in development, diferentiation and maturation of different tissues were further identified. Molecular engineering, mainly recombinant technologies, made bioactive molecules largely available for research and clinical applications. In this issue, Mashayekhi et al. are reviewing the increasing role of engineered bioactive melecules in the treatment of musculoskeletal diseases.

Regenerative medicine (RM) as a novel therapeutical concept is sought to offer revolutionary responses to unmet clinical needs. Making use of cutting edge scientific advancement in the fields of cell and developmental biology, molecular genetics, bioinformatics and biomaterial sciences, regenerative medicine aims tissue and organ restitutio ad integrum. Several technological approaches move RM beyond tissue and organ transplantation, symptomatic pain relief or joint replacement therapies. These approaches include, without being limited to, the use of soluble molecules, gene therapy, stem cell transplantation, tissue engineering and reprogrammed cell pluripotency.

In the early 90 s a Swedish team lead by Brittberg, succesfully peformed the first therapeutical intervention based upon translplanting living cells in a solid tissue [4]. In its initial form, ACT, autologus chondrocyte transplantation, uses adult chondrocytes to treat limited cartilage defects in the knee [4]. A variant of the technique, ChondroCelect was the first Advanced therapy medicinal product (ATMP) to receive marketing authorization in Europe, in October 2010.

Regenerative strategies are increasing in diversity with a large field of potential application for cartilage, bone, menisci and ligament regeneration. Terms like "Biological therapies" or "biological joint resurfacing" are largely vehiculated lately. Their relevance to the orthopedic daily 
practice remains unprecise, mainly due to nonsystematic source of information. In this issue, an elegant review from Kon et al., makes available the latest knowledge about one of the biological therapies with a potential high impact in orthopedic surgery, platelet rich plasma (PRP). Holding extensive experience in cartilage regenerative strategies the authors are explicitly presenting the interest of the procedure for orthopedic aplication as well as the main drawbacks. The need for objective evaluation of PRP and other biological procedures by means of comparative randomized controlled clinical trials (RCT) is stressesd out as a prerogative for future impact and procedure development. Clinical acceptance and of biological therapies will be possible on the extent of quality and quantity of evidence based available medicine data. In this issue, Volz at al are reporting promising medium term results due to efficiency and safety of RCT as compared to the Autologous Matrix Induced Chondrogenesis (AMIC) and microfracture for the treatment of the small and medium size cartilage defects in the knee.

The on-going dialogue between clinical demands, such as science and technology is expanding the panel of therapies for musculoskeletal pathology. Orthopedic practice is constantly increasing in complexity and technicity. Indepth extensive knoweledge and dedicated skills are requiered for the orthopedic practitioner, demanding longer years of training and ultraspecialization. Counteracting the fragmentation of medical practice, systems biology and systems medicine are emerging scientifical and technological breakthroughs with the potential to offer an integrative approach to human health and disease. As proposed by Mashayekhi et al. systems medicine concept and application goes beyond the mere application of systems biology in the medical field. Genomic technologies are consistenly deepening the knowledge of biological compounds, their function and interrelations, in the same time offering the wide unlimited perspective and potential future tools for systemic thinking in orthopedic science and practice. Using extensive omics technologies and systems medicine tools, biomarker discovery in degenerative joint pathology is already begining to deliver tangible results [5]. Other domanins like design of clinical trials, molecular based disease stabilization, personalized approach in drug delivery have the potential of a conssitent improvment in health delivery and prevention in the years to come.

\section{REFERENCES}

[1] Jackson J. Father of the modern hip replacement: Professor Sir John Charnley (1911-82). J Med Biogr 2012; 19 (4): 151-15.

[2] Etghen O, Reginster J-Y. Health related quality of life in total hip and knee arthroplasty. J Bone Joint Surg Am 2004; 86-A (5): 96374.

[3] Urist LMR, Strates BS. Bone Morphogenetic Protein. J Dent Res 1971; 50(6): 1392-406.

[4] Brittberg M, Lindahl A, Nilsson A, Ohlsson C, Isaksson O, Peterson L. Treatment of deep cartilage defects in the knee with autologous chondrocyte transplantation. N Engl J Med 1994; 331(14): 889-95.

[5] Mobasheri A. Osteoarthritis year 2012 in review: biomarkers. Osteoarthr Cartil 2012; 20(12): 1451-64.

(C) Luminita Labusca; Licensee Bentham Open.

This is an open access article licensed under the terms of the Creative Commons Attribution Non-Commercial License (http://creativecommons.org/licenses/by-nc/3.0/) which permits unrestricted, non-commercial use, distribution and reproduction in any medium, provided the work is properly cited. 